\title{
Modular Deformations and Space Curve Singularities
}

Bernd Martin

\begin{abstract}
We investigate different concepts of modular deformations of germs of isolated singularities (infinitesimal, Artinian, formal). An obstruction calculus based on the graded Lie algebra structure of the tangent cohomology for modular deformations is introduced. As the main result the characterisation of the maximal infinitesimally modular subgerm of the miniversal family as flattening stratum of the relative Tjurina module is extended from ICIS to space curve singularities.
\end{abstract}

\section{Introduction}

The notion of a modular deformation has been introduced for complete complex varieties by Palamodov, cf. [P1], later on by Laudal in a formal context, cf. [L], and for analytic polyhedrons in [P3].

The deformation functor of an isolated singularity is usually not universal. Although every deformation of a fixed isolated singularity is induced by a versal deformation of it, the inducing morphism is in general not unique because trivial subfamilies exist in any representing versal family as, for instance, in the case of an isolated complete intersection singularity. One approach to the construction of local moduli for singularities, which should describe continuous invariants in the classification of singularities, is the study of those deformations that do not contain trivial subfamilies. This can be done by restricting the versal family to subgerms that have a universal property at least for all families that they induce.

In [M2] we have used various characterisations of modular families in a strictly local context, i.e., in the category of analytic germs. Here we 
introduce these properties as formal, Artinian and infinitesimal versions of modularity. The associated infinitesimal notion corresponds to injectivity of the relative Kodaira-Spencer map of the deformation. One formal version defined as modular over all Artinian subgerms - we call it Artinian modular - is equivalent to a lifting property of vector fields of the special fiber to vertical vector fields of the family. The equivalence is based on an obstruction calculus for lifting the modularity. The obstruction element is induced by the Lie bracket of the tangent cohomology

$$
T^{0}\left(\mathbf{X}_{\mathbf{0}}\right) \times T^{1}\left(\mathbf{X}_{\mathbf{0}}\right) \longrightarrow T^{1}\left(\mathbf{X}_{\mathbf{0}}\right)
$$

Note that the implication from infinitesimal modularity to modularity is proved for ICIS only with an additional assumption on the singular locus.

The first non-trivial examples of the modular stratum of an isolated complete intersection singularity (ICIS) have been computed in [M2] using a new algorithm to determine the local flattening stratification. Its application is possible by the characterisation of formal modular families of ICIS by flatness of the relative Tjurina module of the deformation. Here we extend this result to space curve singularities.

\section{Modular deformations and their obstructions}

Throughout the paper we will be dealing with analytic germs. Let $\mathbf{X}_{\mathbf{0}}$ be an isolated singularity. Choose a miniversal deformation $F: \mathbf{X} \rightarrow \mathbf{S}$. By definition of versality any other deformation of $\mathbf{X}_{\mathbf{0}}$ over $\mathbf{T}$ is induced from the family $F$, i.e. the functorial maps $\xi_{\mathbf{T}}: \operatorname{Hom}(\mathbf{T}, \mathbf{S}) \longrightarrow \operatorname{Def}_{\mathbf{X}_{\mathbf{0}}}(\mathbf{T})$, $g \mapsto g^{*}(F)$, are all surjective.

Definition 1 A subgerm $\mathbf{M} \subset \mathbf{S}$ of the base space of a miniversal deformation $F: \mathbf{X} \rightarrow \mathbf{S}$ is called modular if for all germs $\mathbf{T}$ the induced maps $\xi_{\mathbf{T}}$ restricted to $\xi_{\mathbf{T}}^{-1}\left(\xi_{\mathbf{T}}(\operatorname{Hom}(\mathbf{T}, \mathbf{M}))\right.$ are injective.

A modular germ is not assumed to be reduced. By definition any two maximal modular subgerms of a miniversal family of $\mathbf{X}_{\mathbf{0}}$ are uniquely isomorphic and independent of the choice of the miniversal family. We call it the modular stratum of the singularity $\mathbf{X}_{\mathbf{0}}$. For instance, the modular stratum of a quasi-homogeneous isolated complete intersection singularity (ICIS) consists of its reduced $\tau$-constant stratum, cf. [A]. However, the modular stratum usually carries a non-reduced structure for most non-quasi-homogeneous hypersurface singularities, cf. [M2].

We have to distinguish carefully between two different versions of the term formally modular. 
Definition 2 A subgerm $\mathbf{M} \subset \mathbf{S}$ of a miniversal deformation $F: \mathbf{X} \rightarrow \mathbf{S}$ is called Artinian modular if any Artinian subgerm $\mathbf{N}$ of $\mathbf{M}$ is modular with respect to $F$.

Any pull-back of an automorphism of $\mathbf{M}$ to the modular family induces an isomorphism of the modular family by definition. Infinitesimally, it corresponds to the statement that any vector field on $\mathbf{M}$ causes a trivial infinitesimal deformation of the modular family. This leads to the infinitesimal notion:

Definition 3 A subgerm $\mathbf{M} \subset \mathbf{S}$ of a miniversal deformation $F: \mathbf{X} \rightarrow \mathbf{S}$ is called infinitesimally modular if the restriction to $\mathbf{M}$ of the relative KodairaSpencer map $\theta_{F}$ of $F$ is injective,

$$
\theta_{F}: T^{0}(\mathbf{S}) \longrightarrow T^{1}(\mathbf{X} / \mathbf{S}), \quad \delta \mapsto c l(\delta(F)) .
$$

Obviously, any modular family is Artinian modular, any Artinian modular family is infinitesimally modular. Are these notions all equivalent as, for instance, in the cited cases considered by Palamodov?

Proposition 4 For a subgerm $\mathbf{M} \subset \mathbf{S}$ of a miniversal deformation $F$ the following statements are equivalent:

(1) $\mathbf{M}$ is infinitesimally modular,

(2) The evaluation map ev $v_{\mid \mathbf{M}}$ from vertical vector fields over $\mathbf{M}$ to vector fields of the special fiber is surjective: $e v_{\mid \mathbf{M}}: T^{0}(\mathbf{X} / \mathbf{S})_{\mid \mathbf{M}} \longrightarrow T^{0}\left(\mathbf{X}_{\mathbf{0}}\right)$,

(3) $\mathbf{M}$ is Artinian modular.

Proof: $(1) \Rightarrow(2)$ This implication follows by a careful analysis of the following long exact sequence — the so called Kodaira-Spencer sequenceof the family $F$ and its evaluation at the special fiber, cf. [P2]:

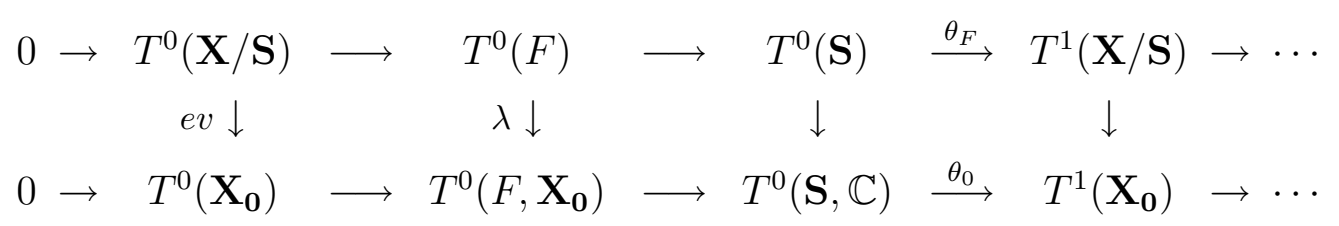

For a miniversal deformation $T^{0}(\mathbf{S}, \mathbb{C})$ is the tangent space of $\mathbf{S}$ and the map $\theta_{0}$ is bijective, hence the image of $\lambda$ lies in $T^{0}\left(\mathbf{X}_{\mathbf{0}}\right)$. Assuming the injectivity of $\theta_{F \mid \mathbf{M}}$ the image of $e v_{\mid \mathbf{M}}$ is equal to that of $\lambda$. Hence, (2) holds if $\lambda$ is surjective. But, this is always fulfilled for a miniversal family $F$ by similar arguments as in [P2, 1.8]. 
Take a vector field $\delta \in T^{0}\left(\mathbf{X}_{\mathbf{0}}\right)$. We want to find a pair of vector fields $\left(\delta^{\prime}, \eta\right) \in T^{0}(F)$, i.e. $\delta^{\prime}$ is a lift of $\eta$ along $F$, and $\delta^{\prime}$ induces $\delta$ at the special fibre. Now $\delta$ gives an automorphism $a_{\delta}$ of $\mathbf{X}_{\mathbf{0}} \times \mathbf{I}$ over $\mathbf{I}:=\operatorname{Spec} \mathbb{C}[\varepsilon]$, which is the identity at the special fibre. Consider the deformation $\tilde{F}:=$ $F \times i d_{\mathbf{I}}: \mathbf{X} \times \mathbf{I} \longrightarrow \mathbf{S} \times \mathbf{I}$. Let $\tilde{F}_{1}$ be the restriction of $\tilde{F}$ to the subspace $\mathbf{T}:=\{\mathbf{0}\} \times \mathbf{I} \cup \mathbf{S} \times\{\mathbf{0}\}$. If $\varphi_{1}: \mathbf{T} \longrightarrow \mathbf{S}$ denotes the obvious map, then $\tilde{F}_{1}=\varphi_{1}^{*}(F)$. Consider the automorphism $\tilde{a}_{\delta}$ of the deformation $\tilde{F}_{1}$ induced by $a_{\delta}$ over the first component of $\mathbf{T}$ and by the identity over the second. Clearly $\tilde{F}_{1}^{\prime}:=\tilde{F}_{1} \cdot \tilde{a}_{\delta}$ is versal itself and the composition $\tilde{a}_{\delta}^{\prime}$ of $\tilde{a}_{\delta}$ with the projection to $\mathbf{X}$ induces the equivalence of the deformations $\tilde{F}_{1}^{\prime}$ and $\varphi_{1}^{*}(F)$.

By versality $\tilde{F}$ is induced from $\tilde{F}_{1}^{\prime}$ by a map $\psi: \mathbf{S} \times \mathbf{I} \longrightarrow \mathbf{T}$. Then $\psi_{1}:=\varphi_{1} \cdot \psi$ is an extension of $\varphi_{1}$ and an extension $\tilde{a}_{1}$ of $\tilde{a}_{\delta}^{\prime}$ to a morphism of deformations exists:

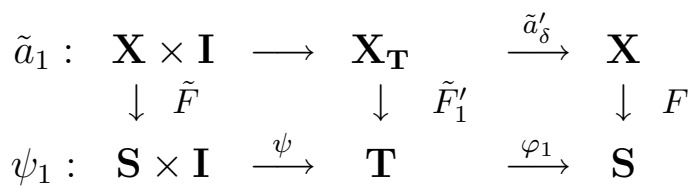

The corresponding homomorphism $\psi_{1}^{*}: \mathcal{O}_{\mathbf{S}} \rightarrow \mathcal{O}_{\mathbf{S}}[\varepsilon]$ induces the vector field $\eta$ by $\psi_{1}^{*}(a)=a+\varepsilon \eta(a)$. Similarly, $\tilde{a}_{1}^{*}$ gives the vector field $\delta^{\prime}$.

$(2) \Rightarrow(3)$ Any Artinian subgerm $\mathbf{A} \subset \mathbf{M}$ is obtained by a finite number of infinitesimally small extensions of the corresponding algebras from $\mathbb{C}$ to $A:=\mathcal{O}_{\mathbf{A}}$. We may consider by induction the previous extension $\eta: 0 \rightarrow$ $K \rightarrow A \rightarrow A_{0} \rightarrow 0$, such that $K=(\varepsilon)$ and $\mathbf{A}_{\mathbf{0}}$ is modular. In order to show that $F$ is modular over $\mathbf{A}$, we need an obstruction calculus for lifting the modularity infinitesimally. First we describe the obstruction element and check its vanishing under assumption (2):

Take any section $s_{0}^{*}: A_{0} \rightarrow A$. Then $F_{A}$ restricted to $\mathbf{A} \times_{s_{0}}\{\mathbf{0}\} \cong \mathbf{I}$ is an infinitesimal deformation $\tilde{f}_{\eta}$. Its class $\operatorname{cl}\left(\tilde{f}_{\eta} \varepsilon\right) \in T^{1}\left(\mathbf{X}_{\mathbf{0}}\right) \otimes K$ does not depend on $s_{0}$. We associate to this class the obstruction element $\mathbf{o}_{F, \eta} \in$ $\operatorname{Hom}\left(T^{0}\left(\mathbf{X}_{\mathbf{0}}\right), T^{1}\left(\mathbf{X}_{\mathbf{0}}\right)\right) \otimes K$, induced by the Lie bracket of the tangent cohomology of $\mathbf{X}_{\mathbf{0}},[-,-]: T^{0} \times T^{1} \longrightarrow T^{1}$, by $\delta \mapsto\left[\delta, c l\left(\tilde{f}_{\eta}\right)\right]$.

In explicit terms the obstruction element $\mathbf{o}_{F, \eta}$ is obtained as follows: Take an embedding of the miniversal family $F: \mathbf{X} \subset \mathbb{C}^{\mathbf{n}} \times \mathbf{S} \longrightarrow \mathbf{S}$. We denote a basis of the defining ideal of $\mathbf{X}$ by $F \in \mathcal{O}_{\mathbb{C}^{\mathbf{n}} \times \mathbf{S}}^{q}$, too, and by $f \in$ $\mathcal{O}_{\mathbb{C}^{\mathbf{n}}}^{q}$ the equations of $\mathbf{X}_{\mathbf{0}}$. Any vector field $\delta \in T^{0}\left(\mathbf{X}_{\mathbf{0}}\right)$ is represented by $\tilde{\delta} \in \operatorname{Der}\left(\mathcal{O}_{\mathbb{C}^{\mathbf{n}}}\right)$, such that $\tilde{\delta}(f)=h f$, where $h \in \operatorname{Mat}\left(q, q ; \mathcal{O}_{\mathbb{C}^{\mathbf{n}}}\right)$. If $\mathbf{A}_{\mathbf{0}} \subset \mathbf{S}$ is modular a lift $\delta_{A_{0}} \in T^{0}\left(\mathbf{X}_{\mathbf{A}_{\mathbf{0}}}, \mathbf{A}_{\mathbf{0}}\right)$ of $\delta$ exists. It is represented by some $\tilde{\delta}_{A_{0}}$, such that $\tilde{\delta}_{A_{0}}\left(F_{A_{0}}\right)=H_{A_{0}} F_{A_{0}}, F_{A_{0}} \in \mathcal{O}_{\mathbb{C}^{\mathbf{n}} \times \mathbf{A}_{\mathbf{0}}}^{q}$ being the class of $F$ modulo the defining ideal of $\mathbf{A}_{\mathbf{0}}, H_{A_{0}} \in \operatorname{Mat}\left(q, q ; \mathcal{O}_{\mathbb{C}^{\mathbf{n}} \times \mathbf{A}_{\mathbf{0}}}\right)$ being a lift of $h$. Choosing a section $s_{0}$ we may write $A=A_{0}[\varepsilon], \varepsilon^{2}=0, F_{A}=F_{A_{0}}+\varepsilon \tilde{f}$, $\tilde{f} \in \mathcal{O}_{\mathbb{C}^{\mathbf{n}}}^{q}$ representing the class $\operatorname{cl}\left(\tilde{f}_{\eta} \varepsilon\right)$ in $T^{1}\left(\mathbf{X}_{\mathbf{0}}\right) \otimes K$. 
Take any representations $\tilde{\delta}_{A}$ and $\tilde{H}_{A}$ over $A$ of $\tilde{\delta}_{A_{0}}$ and $H_{A_{0}}$. One easily checks that $\tilde{\delta}_{A}\left(F_{A}\right)-\tilde{H}_{A} F_{A}$ and $(\tilde{\delta}(\tilde{f})-h \tilde{f}) \varepsilon$ represent the same class in $T^{1}\left(\mathbf{X}_{\mathbf{0}}\right) \otimes K$, being the Lie bracket $[\delta, c l(\tilde{f})] \varepsilon$. Particularly, it is independent of the choices of representations and the section.

By assumption (2) we may choose a lift $\tilde{\delta}_{A} \in T^{0}\left(\mathbf{X}_{\mathbf{A}}, \mathbf{A}\right)$ of $\tilde{\delta}_{A_{0}}$ under $e v$, hence $[\delta, \operatorname{cl}(\tilde{f})] \varepsilon$ and the obstruction element $\mathbf{o}_{F, \eta}$ vanish. By the next Lemma follows that $F$ is modular over $\mathbf{A}$.

In the following we use the description of the obstruction element $\mathbf{o}_{F, \eta}$ from above, which holds for any small extension $\eta: 0 \rightarrow K \rightarrow A \rightarrow A_{0} \rightarrow 0$, $\left(\mathbf{m}_{A} K=0\right)$, of the algebra $A_{0}$ of a modular subgerm. Nevertheless, we may assume that all small extensions are infinitesimal.

Lemma $\mathbf{5}$ Let $F: \mathbf{X} \rightarrow \mathbf{S}$ be a miniversal deformation of $\mathbf{X}_{\mathbf{0}}$, which is modular over an Artinian subgerm $\mathbf{A}_{\mathbf{0}} \subset \mathbf{S}$. Let $\eta: 0 \rightarrow K \rightarrow A \rightarrow A_{0} \rightarrow 0$ be a small extension of the associated Artinian algebra $A_{0}$ inside $\mathcal{O}_{\mathbf{S}}$. Then $F$ is modular over $\mathbf{A}$ iff $\mathbf{o}_{F, \eta}=0$.

Proof: Take any derivation $\delta \in T^{0}\left(\mathbf{X}_{\mathbf{0}}\right)$ and a representation $\tilde{\delta} \in \operatorname{Der}_{A_{0}}\left(\mathcal{O}_{\mathbb{C}}\right.$ $\left.\otimes A_{0}\right)$. It defines an infinitesimal family of automorphisms $a_{\delta} \in A u t_{\mathbf{I}}\left(\mathbb{C}^{\mathbf{n}} \times \mathbf{I}\right)$ by $X_{i} \mapsto X_{i}+\varepsilon \tilde{\delta}\left(X_{i}\right)$. Apply $a_{\delta} \times i d_{\mathbf{A}}$ to $\mathbf{X}_{\mathbf{A}} \times \mathbf{I}$ and get an isomorphic deformation $\mathbf{X}^{\prime}$ :

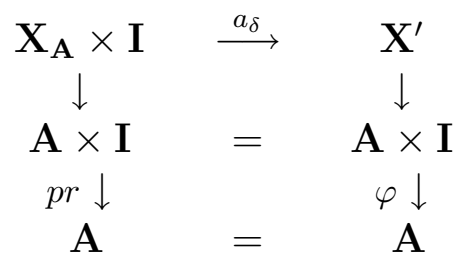

Assume that $F$ is modular over $\mathbf{A}$. Then $\mathbf{X}^{\prime}$ is induced from $F_{A}$ by a unique morphism $\varphi: \mathbf{A} \times \mathbf{I} \rightarrow \mathbf{A}$, such that the above diagram is commutative. Hence, $a_{\delta}$ is an isomorphism over $\mathbf{A}$ and $\mathbf{X}^{\prime}$ is trivial as deformation of $\mathbf{X}_{\mathbf{A}} \rightarrow \mathbf{A}$ over $\mathbf{I}$. We obtain $\left[\delta, \operatorname{cl}\left(\tilde{f}_{\eta}\right)\right]=0$, which implies the vanishing of $\mathbf{o}_{F, \eta}$.

If $\mathbf{A}$ is not modular we may assume that a sub-deformation $\mathbf{Y} \subset \mathbf{X}_{\mathbf{A}} \rightarrow \mathbf{A}$ exists, which has a second and different inducing morphism $\psi$ together with an isomorphism of deformations $a_{0}$ :

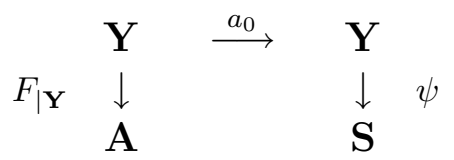

Because $\mathbf{A}_{\mathbf{0}}$ is modular $\psi$ coincides with $F$ over $\mathbf{A}_{\mathbf{0}}$ and we may assume that $a_{0}$ is the identity over $\mathbf{A}_{\mathbf{0}}$. As isomorphism of embedded deformations $a_{0}$ is given by $X_{i} \mapsto X_{i}+\varepsilon \tilde{\delta}\left(X_{i}\right), \tilde{\delta} \in \operatorname{Der}\left(\mathcal{O}_{\mathbb{C}^{\mathbf{n}}}\right)$ representing a vector field $\delta \in T^{0}\left(\mathbf{X}_{\mathbf{0}}\right)$ and $\varepsilon$ given by $A=A_{0}[\varepsilon]$. Because the image of $\psi$ is not contained in $\mathbf{A}$ we have $\left[\delta_{0}, \operatorname{cl}\left(\tilde{f}_{\eta}\right)\right] \neq 0$ and $\mathbf{o}_{F, \eta}$ does not vanish. 
Note that the statements of Proposition 4 remain valid in the category of complete germs and using formal equivalence of deformations as well. The equivalence of modularity and infinitesimal modularity for deformations of complete varieties or analytic polyhedrons, cf. [P1], [P3], is expected to hold in the local case, too. We only show the open implication under an additional assumption. Obviously, an analytic subgerm of the base space of a miniversal deformation, which is formally modular (completions are taken with respect to a section) is modular. We prove the other implication under the following restrictions:

Proposition $\mathbf{6}$ Let $\mathbf{X}_{\mathbf{0}}$ be an ICIS. An analytic subgerm $\mathbf{M}$ of the base space of a miniversal deformation $F$ of $\mathbf{X}_{\mathbf{0}}$, such that any nearby fiber $\mathbf{X}_{\mathbf{t}}$, $t \in \mathbf{M}$, has exactly one singular point, is modular iff it is formally modular.

Proof: By the assumption about the critical locus $F$ has a unique section over $\mathbf{M}$, whose image is the critical locus. We may write $\mathbf{X}_{\mathbf{M}}$ as embedded deformation of $\mathbf{X}_{\mathbf{0}}$ with (zero) section: $\mathcal{O}_{\mathbf{X}_{\mathbf{M}}}=\mathcal{O}_{\mathbf{M}}\{X\} /\left(F_{\mathbf{M}}\right)$. Because $\mathbf{X}_{\mathbf{0}}$ is an ICIS $\mathcal{O}_{\mathbf{X}_{\mathbf{M}}}$ is simultaneously finitely determined over $\mathcal{O}_{\mathbf{M}}$ along the section. Hence two deformations of $\mathbf{X}_{\mathbf{0}}$, such that one is induced from $\mathbf{M}$, are both deformations with section. They are analytically isomorphic iff they are formally isomorphic along the section.

An example — already discussed in [M2] — shows that infinitesimally modular families exist, which admit a splitting critical locus. Consider the following curve singularity, whose equation has a degenerate quasihomogeneous leading form: $\mathbf{X}_{\mathbf{0}}$ defined by $f_{0}=\left(x-y^{3}\right)^{2}\left(x+2 y^{3}\right)+y^{11}$ with Tjurina number $\tau=16$ and Milnor number $\mu=18$. Its deformation $\mathbf{X} \rightarrow \mathbf{T}$ defined by $f(x, y, t)=f_{t}:=f_{0}+t^{2} y^{9}+2 t y^{10}$ over $\mathbf{T}=\mathbb{C}^{1}$ has $\tau\left(f_{t}\right)=15$ and $\mu\left(f_{t}\right)=16$ for $t \neq 0$. Using Singular, cf. [S], one checks that its relative Tjurina algebra $T\left(f_{t}\right):=\mathbb{C}[t]\{x, y\} /\left(f_{t}, \partial_{x} f_{t}, \partial_{y} f_{t}\right)$ is flat over $\mathbb{C}[t]$. Hence the family is infinitesimally modular.

We find two interesting observations: The family $T^{1}(\mathbf{X} / \mathbf{T})$ is not coherent over $\mathbf{T}$, because it is flat, but not free. Moreover, $f_{t}$ has another critical value for $t \neq 0$ at $\left(-t^{3},-t\right)$, hence its global Tjurina number is constant and the critical locus splits. This does not occur for a $\mu$-constant deformation.

Remark 1 The assumption on the critical locus can be interpreted as a kind of finiteness condition: It implies the coherence of $T^{1}(\mathbf{X} / \mathbf{S})_{\mid \mathbf{M}}$, Note, that we lose coherence of the tangent cohomology in a strictly local context. This is avoided in the category of multi-germs. But when doing computations we strictly need the category of analytic or complete local algebras. 


\section{Flatness of the Tjurina module}

The condition of infinitesimal modularity was characterised as a flatness condition of the relative Tjurina module of the deformation for isolated complete intersection singularities, cf. [M2]. This was the main step to apply algorithmic methods for determining modular strata of these singularities. It is not clear at all, whether the statement holds in general or under the slightly weaker assumption as $T^{2}\left(\mathbf{X}_{\mathbf{0}}\right)=0$, which implies that $\mathbf{X}_{\mathbf{0}}$ is unobstructed and $\mathbf{S}$ is smooth. Here we extend the flatness property to the class of reduced space curve singularities, which are not complete intersections.

Let $q$ be the minimal number of generators of the ideal of $\mathbf{X}_{\mathbf{0}} \subset \mathbb{C}^{\mathbf{n}}$ : $I_{0}=\left(f_{1}, \ldots, f_{q}\right) \subset \mathbb{C}\{X\}$. The miniversal family $F$ may be chosen as embedded deformation. Hence the total deformation space is defined by an ideal $I_{\mathbf{X}}=\left(F_{1}, \ldots, F_{q}\right) \subset \mathcal{O}_{\mathbf{S}}\{X\}$. Denote by $J(F)$ the relative Jacobian matrix of $F$ over $\mathbf{S}$ modulo $I_{\mathbf{X}}$ and its cokernel by $\tilde{T}_{\mathbf{X}}:=\mathcal{O}_{\mathbf{X}}^{q} / J(F)$.

Proposition 7 A subgerm $\mathbf{M}$ of the base $\mathbf{S}$ of a miniversal deformation $F$ is infinitesimally modular iff $\tilde{T}_{\mathbf{X}} \otimes \mathcal{O}_{\mathbf{M}}$ is flat over $\mathcal{O}_{\mathbf{M}}$.

Proof: We have the following commutative diagram with exact rows:

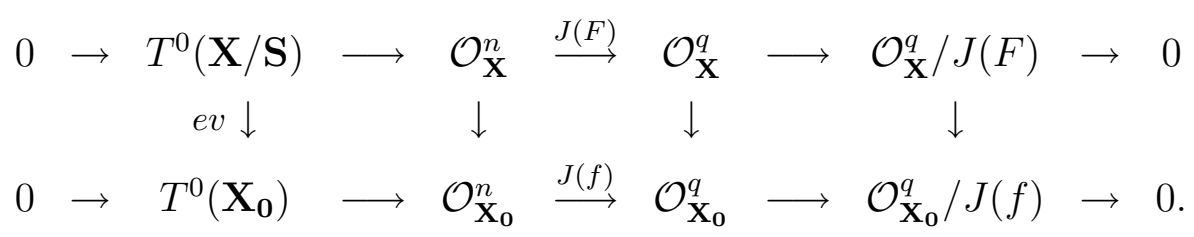

The matrix $J(F)$ is a representation of $\tilde{T}_{\mathbf{X}}=\mathcal{O}_{\mathbf{X}}^{q} / J(F)$ as $\mathcal{O}_{\mathbf{X}}$-module lifting the representation of $\tilde{T}_{\mathbf{X}_{\mathbf{0}}}=\mathcal{O}_{\mathbf{X}_{\mathbf{0}}}^{q} / J(f)$. The module of vertical vector fields $T^{0}(\mathbf{X} / \mathbf{S})$ can be considered as syzygies of the columns of the representation matrices. By Proposition 4 infinitesimal modularity is equivalent to the lifting property of vector fields. The surjectivity of $e v$ over $\mathbf{M}$ means that any syzygy over the special fiber lifts to a syzygy over $\mathbf{M}$. This is exactly a characterisation of flatness, cf. [E, 6.].

We remark that $\tilde{T}_{\mathbf{X}}$ coincides with $T^{1}(\mathbf{X} / \mathbf{S})$ if $\mathbf{X}_{\mathbf{0}}$ is an ICIS.

In the case of space curves singularities, which are unobstructed, we have many informations from its structure as determinantal variety.

Proposition 8 Let $\mathbf{X} \subset \mathbb{C}^{3} \times \mathbf{S} \rightarrow \mathbf{S}$ be a deformation of a reduced space curve singularity $\mathbf{X}_{\mathbf{0}}$ over a smooth germ $\mathbf{S}$, such that the defining ideal $I_{0} \subset \mathcal{O}_{\mathbb{C}}^{3}$ is minimally generated by $q>2$ equations. We have:

(1) A matrix $A \in \operatorname{Mat}(q, q-1 ; \mathcal{O})$ exists, such that $0 \rightarrow \mathcal{O}^{q-1} \stackrel{A}{\longrightarrow} \mathcal{O}^{q} \stackrel{\Delta}{\longrightarrow}$ $\mathcal{O}_{\mathbf{X}} \rightarrow 0$ is exact, $\mathcal{O}:=\mathcal{O}_{\mathbf{S}}\{x, y, z\}$ and $\Delta:=\left(\Delta_{1}, \ldots, \Delta_{q}\right)$ are the $(q-1)$-minors of $A$. 
(2) The relative normal bundle of the deformation $\mathcal{N}_{\mathbf{X}}:=\mathcal{N}_{\mathbf{X}, \mathbb{C}^{3} \times \mathbf{S} \mid \mathbf{S}}$ is represented as $\mathcal{O}_{\mathbf{X}}$-module by a matrix $C \in \operatorname{Mat}\left(q, q^{2}-q ; \mathcal{O}_{\mathbf{X}}\right)$ of $(q-2)$ minors of $A$.

Proof: We obtain (1) because $\mathbf{X}$ is Cohen-Macaulay at codimension two, cf. [St]. The relative normal bundle is represented by the syzygies of the transposed matrix syz $\left(\bar{A}^{t}\right)$ modulo $I_{\mathbf{X}}=(\Delta)$, cf. [M1]. Define the matrix $C$ as follows: The columns of $C$ are indexed by the elements of $A$. The entry of $C$ in the $k$-th row and $(i, j)$-th column is (up to an alternating sign) the $(q-2)$-minor of $A$ obtained by deleting the $k$-th and $i$-th row and the $j$-th column, if $k \neq i$, or 0 otherwise. Let $\mathbf{c}_{i, j} \in \mathcal{O}^{q}$ denote a column of $C$. By Laplace developing we obtain $A^{t} \cdot \mathbf{c}_{i, j}=\Delta_{i} \cdot \mathbf{e}_{j}$, hence $A^{t} \cdot C \equiv 0$ in $\mathcal{O}_{\mathbf{X}}$.

It remains to show that any syzygy $\mathbf{h}$ of $A^{t}$ modulo $I_{\mathbf{X}}$ is contained in the module generated by the columns of $C$ : Over $\mathcal{O}$ we have $A^{t} \cdot \mathbf{h}=$ $\sum_{i, j} H_{i j} \Delta_{i} \mathbf{e}_{j}$, hence $A^{t} \cdot\left(\mathbf{h}-\sum_{i, j} H_{i j} \mathbf{c}_{i, j}\right)=0$. But, $A^{t} \cdot \mathbf{y}=0$ implies that $\mathbf{y}$ is a multiple of $\Delta$ over the quotient field of $\mathcal{O}$. We obtain relatively prime elements $f, g \in \mathcal{O}$, such that $f \mathbf{y}=g \Delta$. This implies that $V(f)$ is contained in $\mathbf{X}$. Because $\mathbf{X}$ is a curve $f$ has to be a unit and $\mathbf{y} \equiv 0$ modulo $I_{\mathbf{X}}$.

Corollary 9 The quotient module $\mathcal{O}_{\mathbf{X}}^{q} / \mathcal{N}_{\mathbf{X}}$ is flat over $\mathcal{O}_{\mathbf{S}}$.

Proof: The quotient module is represented as $\mathcal{O}_{\mathbf{X}}$-module by the matrix $A^{t}$. At the special point $\mathbf{0} \in \mathbf{S}$ the module $\mathcal{O}_{\mathbf{X}_{\mathbf{0}}}^{q} / \mathcal{N}_{\mathbf{X}_{\mathbf{0}}}$ is represented by the matrix $A_{0}^{t}:=A^{t}$ modulo $\mathrm{m}_{\mathbf{S}}$. Their syzygies are generated by the columns of the matrices $C$ resp. $C_{0}$ given by minors of $A$ resp. $A_{0}$ in the same way. Hence any syzygy of $A_{0}$ may be lifted.

Proposition 10 Let $F: \mathbf{X} \rightarrow \mathbf{S}$ be a miniversal deformation of a reduced space curve singularity. Then $\mathbf{M} \subset \mathbf{S}$ is infinitesimally modular iff $T^{1}(\mathbf{X} / \mathbf{S})_{\mid \mathbf{M}}$ is flat as $\mathcal{O}_{\mathbf{M}}$-module.

Proof: By Proposition 7 we have to show that $\tilde{T}_{\mathbf{X}} \otimes \mathcal{O}_{\mathbf{M}}$ is flat iff $T^{1}(\mathbf{X} / \mathbf{S})_{\mid \mathbf{M}}$ is flat. But, $T^{1}(\mathbf{X} / \mathbf{S})$ is the cokernel of

$$
\Theta_{\mathbb{C}^{3} \times \mathbf{S}} \otimes \mathcal{O}_{\mathbf{X}} \stackrel{J(F)}{\longrightarrow} \mathcal{N}_{\mathbf{X}}
$$

cf. [M1, 3.1]. The statement follows from the short exact sequence

$$
0 \rightarrow T^{1}(\mathbf{X} / \mathbf{S}) \rightarrow \tilde{T}_{\mathbf{X}} \rightarrow \mathcal{O}_{\mathbf{X}}^{q} / \mathcal{N}_{\mathbf{X}} \rightarrow 0
$$

by Corollary 9 . 


\section{References}

[A] Aleksandrov, A. G.: Cohomology of a quasihomogeneous complete intersection. Izv. Akad. Nauk SSSR Ser. Mat. 49 (1985), 467-510.

[E] Eisenbud, D.: Commutative Algebra with a View Towards Algebraic Geometry. Springer-Verlag, Berlin, New York, 1996.

[L] Laudal, O. A.: Formal moduli of algebraic structures. Lecture Notes in Math. 754, Springer-Verlag, Berlin-New York, 1979.

[M1] Martin, B.: Computing Versal Deformations with Singular. In Algorithmic Algebra and Number Theory (Heidelberg, 1997), 283-294. SpringerVerlag, Berlin-New York, 1998.

[M2] Martin, B.: Algorithmic Computation of Flattenings and of Modular Deformations. J. Symb. Comp. 34 (2002), 199-212.

[P1] Palamodov, V.P.: Moduli and versal deformations of complex spaces. In Variétés analytiques compactes (Colloq., Nice, 1977), 74-115. Lecture Notes in Math. 683, Springer, Berlin, 1978.

[P2] Palamodov, V.P.: Tangent fields on deformations of complex spaces. Math. USSR-Sb. 71 (1992), no. 1, 163-182.

[P3] Palamodov, V.P.: Deformation of analytic polyhedrons. J. Algebraic Geom. 2 (1993), 263-294.

[S] Greuel, G.-M., Pfister, G., Schönemann, H.: Singular 2.0. A Computer Algebra System for Polynomial Computation. Centre for Computer Algebra, Univ. of Kaiserslautern, 2001. http://www . singular .uni-kl.de

[St] Van Straten, D.: A note on the discriminant of a space-curve. Manuscripta Math. 87 (1995), 167-177.

Recibido: 4 de abril de 2002

Revisado: 24 de enero de 2003

Bernd Martin

Institut für Mathematik

BTU Cottbus, PF 101344

D 03013 Cottbus, Deutschland

martin@math.tu-cottbus.de

Partially supported by G.I.F. 